\title{
TiN Coated Aluminum Electrodes for DC High Voltage Electron Guns
}

\author{
M.A. Mamun, A. A. Elmustafa ${ }^{\text {a) }}$ \\ Old Dominion University, Norfolk, VA 23529, USA \\ The Applied Research Center, Thomas Jefferson National Accelerator Facility, Newport News, VA \\ 23606, USA \\ R. Taus \\ Loyola Marymount, Los Angeles, CA 90045, USA \\ E. Forman, M. Poelker \\ Thomas Jefferson National Accelerator Facility, Newport News, VA 23606, USA \\ a)Electronic mail: aelmusta@odu.edu
}

\section{ABSTRACT}

Preparing electrodes made of metals like stainless steel, for use inside DC high voltage electron guns, is a labor-intensive and time-consuming process. In this paper we report the exceptional high voltage performance of aluminum electrodes coated with hard titanium nitride (TiN). The aluminum electrodes were comparatively easy to manufacture and required only hours of mechanical polishing using silicon carbide paper, prior to coating with TiN by a commercial vendor. The high voltage performance of three TiNcoated aluminum electrodes, before and after gas conditioning with helium, was compared to that of bare aluminum electrodes, and electrodes manufactured from titanium alloy (Ti-6Al-4V). Following gas conditioning, each TiN-coated aluminum electrode reached $-225 \mathrm{kV}$ bias voltage while generating less than $100 \mathrm{pA}$ of field emission (<10 pA) using a $40 \mathrm{~mm}$ cathode/anode gap, corresponding to field strength of 
13.7 MV/m. Smaller gaps were studied to evaluate electrode performance at higher field strength with the best performing TiN-coated aluminum electrode reaching $\sim 22.5 \mathrm{MV} / \mathrm{m}$ with field emission less than $100 \mathrm{pA}$. These results were comparable to those obtained from our best-performing electrodes manufactured from stainless steel, titanium alloy and niobium, as reported in references cited below. The TiN coating provided a very smooth surface and with mechanical properties of the coating (hardness and modulus) superior to those of stainless steel, titanium-alloy and niobium electrodes. These features likely contributed to the improved high voltage performance of the TiN-coated aluminum electrodes. 


\section{INTRODUCTION}

Particle accelerators are important tools used in many applications ranging from cancer therapy and industrial linear accelerators and synchrotron light sources, to high energy colliders that are used to investigate nature's fundamental building blocks and forces. The performance of many present and future particle accelerators is limited by the maximum operating voltage supported by the direct current (DC) high voltage photoelectron gun that provides the electron beam for the accelerator. Too often, the operating voltage of the photogun is limited by field emission from the cathode electrode which degrades vacuum via electron stimulated desorption leading to reduced photoelectron yield (or quantum efficiency, QE) via ion bombardment. At high levels (> $1 \mathrm{uA}$ ), field emission can open flange joints and damage high voltage insulators. Cathode electrodes for electron guns are typically manufactured from physically hard metals like stainless steel, titanium and molybdenum ${ }^{1-4 .}$ Table I lists common electrode materials, with relevant properties. Once fabricated at the machine shop, the relatively rough surface of the electrode must be mechanically polished by hand using silicon carbide paper and diamond grit, to obtain a smooth surface free of protrusions ${ }^{5}$. This is a timeconsuming and labor-intensive process. At least one photogun group has implemented electropolishing as a means to speed electrode preparation ${ }^{6,7}$, however our recent tests indicate electropolished electrodes do not respond to gas conditioning as favorably as mechanically polished electrodes ${ }^{8,9}$. Niobium electrodes perform well at high voltage when surfaces are etched with a mixture of strong acids - a technique known as buffered chemical polishing $(\mathrm{BCP})^{10}$. The $\mathrm{BCP}$ process significantly reduces electrode preparation time but not everyone has access to BCP facilities. 
Another important consideration is effective heat removal from the photocathode during high current applications. Only a small portion (few percent) of absorbed laser light produces the desired electron beam. Most of the light simply serves to heat the photocathode and cathode electrode. For high current applications, where many Watts of laser light are required, it is important to maintain the photocathode at temperatures below some limit to ensure that the photocathode does not decompose. Hence, the heat must be removed, which is a complicated task because the cathode is biased at high voltage. Passive heat removal is preferred.

Bare aluminum is not considered a good candidate material for high voltage electrodes. Bare aluminum electrodes - with soft, porous and oxidized surfaces - exhibit high levels of field emission at modest voltages and field strengths, as we illustrate below. However, aluminum is easy to machine and polish, and it provides a high degree of thermal conductivity which could be exploited for passive heat removal from the photocathode, for high current applications. The goal of this work was to evaluate the performance of bare aluminum electrodes coated with titanium nitride, a commerciallyavailable coating commonly used to harden the surface of metals, and with significantly higher work function compared to other materials. The performance of three TiN-coated aluminum electrodes was compared to that of bare aluminum electrodes, and electrodes manufactured from titanium-alloy (Ti-6Al-4V). The titanium-alloy electrodes provide a benchmark-level of performance, representative of mechanically polished electrodes prepared in a traditional manner with silicon-carbide paper and diamond-polishing paste. As presented below, the TiN-coated aluminum electrodes performed significantly better than bare aluminum electrodes, and exhibited performance comparable to the Ti-alloy 
electrodes. All of the TiN-coated aluminum electrodes reached $-225 \mathrm{kV}$ with cathode/anode gap of $50 \mathrm{~mm}$ without measureable field emission $(<10 \mathrm{pA})$, following gas conditioning using helium. The best TiN-coated aluminum electrode exhibited less than $100 \mathrm{pA}$ of field emission at $22.5 \mathrm{MV} / \mathrm{m}$.

The Fowler-Nordheim theory of field emission suggests that smooth electrode surfaces, made of materials with a large work function, exhibit smaller levels of field emission. However, in practice, few people use the Fowler-Nordhiem theory to predict the onset of field emission from individual test samples. It is not uncommon for nominally identical electrodes to exhibit markedly different performance. Part of the problem in predicting the performance of an electrode is that DC field emission involves a complex mixture of macroscopic and microscopic effects, which are determined by the geometry, metallurgy, and surface preparation techniques which can introduce contamination. In this work, gas conditioning was implemented as a means of process control $^{20}$ to minimize the variability of results between nominally identical electrode samples, and to provide a significantly more reliable assessment of high voltage performance. For most of the work reported here, helium was used for gas conditioning, but when stubborn field emitters were encountered, krypton gas was sometimes used.

Excessive field emission often leads to permanent damage on the electrode surface. Therefore, the surface topography of each electrode was investigated at every step of the polishing process, the coating process, and following each step of the high voltage evaluation, using a JEOL JSM-6060 LV scanning electron microscope (SEM). In addition, the surface roughness of the aluminum electrode was inferred based on measurements of small test coupons $(2 \mathrm{~cm} \times 2 \mathrm{~cm} \times 0.5 \mathrm{~cm})$, before and after TiN 
coating, using a Digital Instruments Dimension 3100 atomic force microscope (AFM) in the tapping mode. It was anticipated that a harder electrode surface was less prone to surface damage due to handling and HV processing. Thus, the mechanical properties of the TiN coating, hardness and modulus, were evaluated using nanoindentation measurement, and compared to those of stainless steel, niobium and titanium-alloy coupons. A Nanoindenter XP from Agilent Technologies (Agilent Technologies, Inc., Santa Clara, CA) equipped with a three-sided diamond Berkovich indenter tip was employed to test the coupons in Continuous Stiffness Measurement (CSM) protocol.

\section{EXPERIMENT DESCRIPTION A. Apparatus}

A total of seven electrodes were evaluated: two bare aluminum electrodes, three TiN-coated aluminum electrodes, and two Ti-alloy electrodes. Two of the TiN-coated aluminum electrodes were first evaluated in the uncoated state. Each electrode had a Pierce-type geometry with $25^{\circ}$ focusing angle $(6.35 \mathrm{~cm}$ diameter, $2.85 \mathrm{~cm}$ thick) [Fig. 1(a)], identical to electrodes used at the Continuous Electron Beam Accelerator Facility (CEBAF) for many years ${ }^{21}$. A piece of polished stainless steel was used in place of the GaAs photocathode.

Electrodes were evaluated using two vacuum test chambers TS1 and TS2 [Fig. 1(b) and (c)], identical in function, but with different vacuum pumping technology. One chamber (TS1) relied on non-evaporable getter pump (SAES GP500) and a $220 \mathrm{~L} \mathrm{~s}^{-1}$ ion pump. After installing a test electrode, the entire vacuum chamber was baked at $200^{\circ} \mathrm{C}$ for 30 hours to eliminate water from the vacuum chamber and provide partial activation 
of the non-evaporable getter pump. The base pressure inside this test chamber was $1 \times 10^{-11}$ Torr $\left(1.33 \times 10^{-9} \mathrm{~Pa}\right)$ during electrode evaluation. The other test stand (TS2) was pumped using a cryopump (CTI, $2000 \mathrm{~L} \mathrm{~s}^{-1}$ rated pumping speed) and $50 \mathrm{~L} \mathrm{~s}^{-1}$ ion pump. An electrode was installed, the system was pumped using a small turbo, and then the valve to the cryopump opened. The system was allowed to pump overnight. The next day, the electrode was heated to $200^{\circ} \mathrm{C}$ for eight hours using a smaller heater inserted into the tapered opening of the high voltage insulator to liberate loosely bound adsorbed gasses from the insulator and electrode surfaces. The base pressure in this test stand during electrode evaluation was typically $\sim 1 \times 10^{-10}$ Torr $\left(1.33 \times 10^{-8} \mathrm{~Pa}\right)$. A comparison of the field emission characteristics of three electrodes evaluated in both test stands indicated that field emission results were independent of the vacuum test chambers as long as the vacuum preparation protocols and degassing of the electrodes were conducted in a consistent manner for each chamber.

Details of the test apparatus can be found in ref $^{10}$, an abbreviated description is provided here. Cathode electrodes were suspended from a tapered conical insulator that extended into each test chamber [Fig. 1]. The anode was made from a stainless steel (304L) flat plate with a Rogowski edge profile, polished using 600 grit silicon carbide paper, electrically isolated from ground and attached to a sensitive current meter (Keithley electrometer model 617). The anode could be moved up or down to vary the cathode/anode gap and therefore the field strength. Further details of the cathode electrodes geometry, gap separation, and maximum field strength can be found in ref ${ }^{10}$.

A $-225 \mathrm{kV}$ commercial high voltage power supply was used for the experiment. The high voltage power supply and the ceramic insulator accommodate "industry 
standard" high voltage cables with R-28 connectors. A $100 \mathrm{M} \Omega$ conditioning resistor was placed in series with the cathode electrode via an oil tank and served to protect the apparatus in case of sudden discharge of stored energy. The resistor also serves to protect the electrode via a negative feedback mechanism - as current increases, a larger voltage drop occurs across the resistor, reducing voltage at the electrode.

An assessment of the field emission properties of each test electrode involved monitoring vacuum level inside the apparatus, $x$-ray radiation near the apparatus, and anode current while increasing the voltage applied to the cathode electrode. High voltage was first applied to the electrode using the largest cathode/anode gap of $50 \mathrm{~mm}$. Upon successful high voltage processing (defined below), the gap could be decreased to achieve higher field strength. However, small gaps sometimes produced catastrophic breakdown and electrode damage, so small gap evaluation was approached with caution, and only if very little field emission was observed at a larger gap of $20 \mathrm{~mm}$.

Electrodes were first evaluated under the best vacuum conditions each vacuum chamber could possibly provide. Voltage was applied to the electrode and was gradually increased while maintaining the anode current less than $~ 100$ nanoamperes $(\mathrm{nA})$. As the voltage was increased, field emission would be observed, and sometimes field emission sites would "burn off", as evidenced by lower levels of current measured at the anode. Frequently, stubborn field emitters were encountered, and gas conditioning was employed $^{1}$. Gas conditioning involved introducing helium into the vacuum chamber at pressure between $1 \times 10^{-6}$ to $1 \times 10^{-4}$ Torr $\left(1 \times 10^{-4}\right.$ to $\left.1 \times 10^{-2} \mathrm{~Pa}\right)$, while the cathode electrode was biased at a voltage high enough to produce significant levels of field emission $(\sim$ few $\mu \mathrm{A})$. Under these conditions, the inert gas can be ionized by the field emission and these 
ions are then attracted to the negatively biased cathode electrode, bombarding the electrode in the vicinity of the field emitter. Recent work has revealed that field emission sites are eliminated via two mechanisms ${ }^{20}:$ sputtering and ion implantation which serves to increase the localized work function. Gas conditioning typically was performed for 30 to 60 minutes and repeated multiple times, depending on the performance of the test electrode in the field emission reduction process. An electrode was considered fully processed when field emission current was stable to within a few percent of the average value. Specific details on how to implement gas conditioning are given in ref $^{10,20}$. During gas conditioning, ion pumps were not energized, and the valve to the cryopump was closed. NEG pumps do not pump inert gas. An overboard turbo pump was used to remove the gas from each system, during gas conditioning, and for approximately 30 minutes once the supply of gas was terminated. The pressure inside the vacuum chamber typically recovered to nominal static vacuum level in just 24 hours.

\section{B. Polishing and Preparing the electrodes}

Two electrodes were manufactured from Ti-6Al-4V titanium alloy, and represent the "hard metal" benchmark for comparing the performance of "soft metal" aluminum and TiN-coated aluminum electrodes. The Ti-alloy electrodes were cut to shape using hydrocarbon-free lubricants, and polished on a potter's wheel with silicon carbide paper of increasingly finer grit (300 and then 600 particles/in ${ }^{2}$ ) followed by polishing with diamond grit $(6 \mu \mathrm{m}, 3 \mu \mathrm{m})$, to obtain a mirror-like finish. Between each polishing step, the electrodes were cleaned in an ultrasonic bath using a detergent alkali solution. After 
mechanical polishing, the electrodes were high-pressure rinsed (1200 psi) for 20 minutes with ultrapure de-ionized water with resistivity $>18 \mathrm{M} \Omega \mathrm{cm}$.

Aluminum electrodes were manufactured from Al6061alloy. After being cut to shape with hydrocarbon-free lubricants, each electrode was polished on a potter's wheel with silicon carbide paper of increasingly finer grit: 400, 600, and 800 particles/in ${ }^{2}$. Two electrodes were further polished using 1200 particles/in ${ }^{2}$ paper (a minor goal was to determine if polishing with 1200 grit paper was necessary). Polishing with silicon carbide paper produced a mirror-like, sub-micron surface finish. Between each polishing step, the electrodes were cleaned in ultrasonic bath of acetone and then methanol. Aluminum electrodes required only hours of mechanical polishing using silicon carbide paper, compared to weeks of polishing time for relatively harder materials like stainless steel and titanium alloy.

Two bare aluminum electrodes were tested at high voltage. Afterwards these electrodes received additional polishing to remove defects introduced as a result of field emission and gas conditioning, as described below in section III A. A total of three barealuminum electrodes were then sent to the commercial vendor Beam Alloy Technology LLC for coating with titanium nitride, using an ion-beam enhanced deposition process at low temperature $\left(<93{ }^{\circ} \mathrm{C}\right)^{22}$. According to the vendor, the independently-controlled ion beam provides precise control of film growth, and results in a tight metallurgical bond between the coating and the substrate material. The coating thickness was controlled to $2.5 \mu \mathrm{m}$. Upon return, the electrodes were cleaned in an ultrasonic bath of methanol.

\section{HIGH VOLTAGE TEST RESULTS}


The evaluation of seven test electrodes at high voltage required several months of effort. Similar to past studies ${ }^{10,20}$, it was not uncommon to observe completely different field emission behavior from nominally identical test samples, upon the first application of high voltage. The variability in results associated with initial high voltage processing of electrodes of the same material and treatment, was attributed to undetectable levels of contamination and/or sub-surface variations from sample to sample. Credible results were typically obtained subsequent to gas conditioning, which represented an important aspect of the process control. With some exceptions, most of the results presented below represent post-gas conditioning results.

In Fig. 2, field emission current for a subset of the electrodes, is plotted versus the applied voltage and field strength. The error bars represent one standard deviation in this figure. The field strength for each gap setting was estimated using the electrostatic field mapping program POISSON ${ }^{23}$. Field strength values required to produce $100 \mathrm{pA}$ of field emission, for different cathode/anode gaps, and for all electrodes, are shown in Table II. All values correspond to performance following gas conditioning, with the exception of titanium-alloy sample 2, which exhibited small levels of field emission without gas conditioning. Entries with greater-than sign (>) indicate the electrode did not produce 100 pA of field emission for that gap setting.

\section{A. HV characterization results of bare aluminum electrodes}

Two uncoated aluminum electrodes were evaluated at high voltage, before and after helium gas conditioning. Because aluminum is so soft, helium was chosen over heavy gas species like krypton which can actually create field emission sites, due to a 
comparatively enhanced sputtering rate. In contrast, the benefits of helium gas conditioning stem largely from implantation, rather than sputtering. Based on previous work, helium gas conditioning was shown to be more effective at suppressing field emission when the test sample was biased at lower voltages and for smaller cathode/anode gaps - conditions that ensure ions are implanted at shallow depths, thereby serving to most effectively increase the work function of the surface. After helium gas conditioning, electrodes were re-characterized under the best vacuum conditions. A representative collection of results is shown in Fig. 2(a), for one bare-aluminum test electrode, corresponding to sample A1 in Table II.

Before helium gas conditioning, bare aluminum electrode A1 exhibited 100 pA of field emission at voltages between -64 and $-36 \mathrm{kV}$, for cathode/anode gaps between 50 and $20 \mathrm{~mm}$, corresponding to field strengths of 3.0 and $3.6 \mathrm{MV} / \mathrm{m}$. Bare aluminum electrode sample A2 performed better, with 100 pA of field emission observed at voltages between -90 and $-65 \mathrm{kV}$ and field strengths 5 and $5.4 \mathrm{MV} / \mathrm{m}$, for the same range of cathode/anode gaps. The high voltage performance of both electrodes improved significantly upon helium gas conditioning, with 100 pA of field emission observed at voltages between -158 and $-121 \mathrm{kV}$ and field strengths between 8.5 and $12.0 \mathrm{MV} / \mathrm{m}$. However, it must be noted that for both bare aluminum electrodes, improved performance could be short lived. Gas conditioning could once again be implemented to minimize field emission, but the back and forth process between poor and improved performance would repeat, sometimes because voltage was pushed too high, but also sometimes without warning, with high levels of field emission observed at a voltage that earlier provided zero field emission current. 


\section{B. HV characterization results of TiN coated aluminum electrodes}

The three TiN-coated aluminum electrodes, polished with 1200 and 800 grit silicon carbide paper, exhibited dissimilar high voltage performance prior to helium gas conditioning, however, they behaved similarly afterwards. Only the post-gas conditioning results are shown in Fig. 2(b), which shows the performance of two TiN-coated electrodes. The results for the third TiN-coated aluminum electrode were similar, but omitted from the graph to provide clarity. Field emission current did not reach $100 \mathrm{pA}$ even at the maximum voltage of $-225 \mathrm{kV}$ for cathode/anode gaps $30 \mathrm{~mm}$ and larger. This clearly represents a significant improvement over the uncoated-aluminum electrode performance. For cathode/anode gaps in the range of 30 to $20 \mathrm{~mm}, 100 \mathrm{pA}$ of field emission was observed at voltages between -222 and $-212 \mathrm{kV}$, corresponding to field strength between 15.1 and $18.4 \mathrm{MV} / \mathrm{m}$.

TiN-coated aluminum electrode sample TN2 was studied further in the baked high voltage test stand, TS1, which could accommodate cathode/anode gaps smaller than $20 \mathrm{~mm}$, and hence subjecting the electrode to higher field strength. These data are plotted in Fig. 2(b), with suffix designation "B" for baked. No measurable field emission current was observed $(<10 \mathrm{pA})$ at the maximum voltage of $-225 \mathrm{kV}$ for gap settings $20 \mathrm{~mm}$ and larger. The electrode produced less than $15 \mathrm{pA}$ of field emission current at $-175 \mathrm{kV}$ for a $10 \mathrm{~mm}$ cathode/anode gap which corresponds to a field strength of $22.5 \mathrm{MV} / \mathrm{m}$. This level of performance was achieved without gas conditioning. At higher voltage, breakdown was observed, i.e., field emission current suddenly went from small to large, 
and the field emitter(s) could not be processed-out via gas conditioning. Upon inspection, the electrode possessed a noticeable visual defect. Breakdown occurred at a voltage consistent with the predictions of $\mathrm{P}$. Slade, as shown in reference $\mathrm{e}^{23}$.

Of the three TiN-coated aluminum electrodes, sample TN2 performed the best, reaching the highest voltages and field strengths while exhibiting the smallest levels of field emission. But as Table II suggests, the other TiN-coated aluminium electrode samples performed similarly. The other samples (TN1 and TN3) were pushed to higher voltages and field strengths similar to sample TN2 to evaluate coating limitations. Although neither of the other two samples suffered breakdown, they both suffered reduced performance that could not be recovered with further gas conditioning, at least not as it was implemented. At this point, field emission was observed from TiN-coated electrode samples TN1 and TN3 at voltages and field strengths approximately $50 \%$ of values as indicated in Fig. 2(b) and Table II. Upon inspection, no obvious defects were visible to the naked eye, or using a microscope.

\section{HV characteristics results of diamond paste polished Ti-alloy electrodes}

Two Ti-alloy electrodes were painstakingly polished to a mirror like surface finish using diamond grit and characterized at high voltage [Fig. 2(c)]. As often happens when evaluating nominally identical electrodes, dissimilar performance was observed for the two Ti-alloy electrodes, but in this case, dissimilar performance was maintained even after gas conditioning. Before gas conditioning, Ti-alloy electrode sample TA1 generated $100 \mathrm{pA}$ of field emission at voltages between -192 and $-153 \mathrm{kV}$ for cathode/anode gaps 
between 50 and $20 \mathrm{~mm}$, corresponding to field strengths of 10.9 and $13.0 \mathrm{MV} / \mathrm{m}$. This performance is comparable to that of traditional diamond-paste polished stainless steel ${ }^{10}$. Ti-alloy electrode sample TA1 was subjected to helium gas conditioning, which actually resulted in degraded performance. The post-helium gas conditioning data are not included in Fig. 2(c).

In sharp contrast, Ti-alloy electrode sample TA2 performed far superior to sample TA1, exhibiting less than $20 \mathrm{pA}$ of field emission at the maximum voltage $-225 \mathrm{kV}$ for cathode/anode gaps between 20 and $50 \mathrm{~mm}$. This electrode was studied at higher field strengths using smaller gaps of 17,14 , and $11 \mathrm{~mm}$, where $100 \mathrm{pA}$ of field emission was observed at voltages between -200 and $-153 \mathrm{kV}$, corresponding to field strengths of 18.4 and $21.2 \mathrm{MV} / \mathrm{m}$.

\section{IV.SURFACE EVALUATION}

\section{A. Topography and chemical composition}

Surface characterization of the electrodes was performed using a Joel 6060LV Scanning Electron Microscope (SEM) with $3 \mathrm{~nm}$ resolution. Surface images for an aluminum electrode polished with 1200 grit silicon carbide paper are shown in Fig. 3. Tooling marks could be seen on the polished, uncoated bare aluminum electrode surface [Fig. 3(a)], which exhibited numerous defects including pits and protrusions. No evidence of residual silicon carbide particulate contamination was observed. It is reasonable to assume tooling marks and defects represent potential field emission sites. Gas conditioning of the bare aluminum electrode introduced additional defects, like the one displayed in Fig. 3(b). So although, helium gas conditioning served to reduce overall field 
emission from both of the bare aluminum electrodes that were studied, ion bombardment and surface sputtering lead to significant transformation of surface topography.

The TiN coating served to smooth the bare aluminum electrode surface, concealing defects like the one barely visible in the center of the photo in Fig. 3(c). No change in TiN coating surface morphology was observed, before and after gas conditioning. The image in Fig. 3(d) depicts a damaged TiN coating that resulted when TiN-coated aluminum electrode sample TN2 was exposed to very high field strength, incurring breakdown.

The surface morphology and film thickness was characterized using a 2D surface profilometer and an AFM microscopy on small coupons representing prior and post coated electrode surfaces. The RMS surface roughness of 20 and $16 \mathrm{~nm}$ for the 800 and 1200 grid polished bare aluminum samples is measured from a $2 \mathrm{~mm}$ line scan using the profilometer. The surface roughness decreased from $16 \mathrm{~nm}$ for prior coating of the TiN coated aluminum sample (1200 grit polished) to $13 \mathrm{~nm}$ post coating. Two and three dimensional AFM images of the surface morphology of the TiN coating on the 1200 grit polished coupon is presented in Fig. 4. From a scan area of $1 \mu \mathrm{m} \times 1 \mu \mathrm{m}$, the average values of the RMS roughness and grain height were measured as 7 and $20 \mathrm{~nm}$, respectively. These independent measurements of the roughness (using profilometer and atomic force microscope), are reasonably consistent and indicate a very smooth TiN coating, smoother than metal surfaces that are polished with diamond paste, or chemically etched ${ }^{10}$. The film thickness of the TiN coating on a 1200 grit polished $\mathrm{Al}$ electrode was also measured as $\sim 2.5 \mu \mathrm{m}$ using a step-height profilometry. 


\section{B. Hardness measurement}

Nanomechanical properties of aluminum test coupons were evaluated, before and after the TiN coating. Nanoindentation experiments were performed using an $\mathrm{XP}$ nanoindenter from Agilent in conjunction with the continuous stiffness method (CSM) in depth control mode to measure the hardness of the TiN thin films. Using the CSM technique, the hardness was continuously measured as a function of penetration depth into the surface by superimposing a small harmonic force oscillation (usually resulting in a harmonic displacement oscillation of $2 \mathrm{~nm}$ or less) on the tip during the loading cycle. This allowed the stiffness of contact, and subsequently the mechanical properties of the sample to be constantly evaluated by analyzing the harmonic force and harmonic displacement data, as detailed elsewhere ${ }^{24,25}$. A total of 15 indents with maximum indentation depth of $1 \mu \mathrm{m}$ were performed on each sample. The allowable drift rate and the strain rate for loading were specified as $0.05 \mathrm{~nm} / \mathrm{s}$ and $0.05 \mathrm{~s}^{-1}$, respectively.

The results are indicated in Fig. 5, where the hardness and modulus are plotted versus contact depth. The TiN-coating hardness and modulus were 18 and $270 \mathrm{GPa}$, respectively, at $50 \mathrm{~nm}$ depth of indentation. In comparison, the hardness and modulus of bare aluminum was, 2 and $70 \mathrm{GPa}$, respectively. Clearly, the TiN coating served to harden the surface. Hua and $\mathrm{Li}[\mathrm{x}]$ concluded that the mechanical properties such as the yield strength, modulus, and hardness strongly affect the work function to the sixth power. Based on the dependence of the elastic modulus on the electron work function and the Peierls-Nabarrow model, they also correlated the yield strength and hardness of metals with the electron work function and concluded that the established intrinsic sixthpower correlations are consistent with reported experimental measurements ${ }^{26}$. In general, 
electrodes with higher work function are anticipated to demonstrate higher high voltage standoff and therefore TiN-coated aluminum electrodes with superb hardness and elastic modulus likely lead to enhanced electron work function.

To qualitatively assess the adhesion of the TiN coating to the bare aluminum, SEM microscopy scans were conducted on the residual impressions left by the indenter, for different depths of indentation, Fig. 6. The images of $500 \mathrm{~nm}$ to $3 \mu \mathrm{m}$ deep Berkovich indents on the TiN coated 1200 grit polished aluminum sample indicate no signs of delamination or peeling of the film from the substrate. However, non-radial fracture mode was observed for the larger indents [Fig. 6(b)]. For a hard-on-soft film system like TiN on A16061, the film experienced successive fracture events at indentation depths larger than $500 \mathrm{~nm}$, which was observed as pop-in events during the loading cycle of indentation test, as depicted in Fig. 7.

\section{SUMMARY}

We have shown that aluminum electrodes, coated with TiN, can be used inside DC high voltage electron guns. The aluminum electrodes required less time and money to prepare, compared to electrodes manufactured from harder materials. The application of the hard TiN coating serves to protect the soft aluminum electrode from scratching and produces a surface finish superior to a diamond-paste polished surface. Also when biased at high voltage and exposed to high field strength, the TiN-coated aluminum electrodes exhibited comparatively low levels of field emission. All three TiN-coated aluminum electrodes reached $-225 \mathrm{kV}$ without field emission at $50 \mathrm{~mm}$ cathode/anode gaps, corresponding to field strength of $\sim 13.0 \mathrm{MV} / \mathrm{m}$. The best performing TiN-coated 
aluminum electrode produced $100 \mathrm{pA}$ of field emission at a field strength of $22.5 \mathrm{MV} / \mathrm{m}$. We attribute the superb performance of the coated electrodes to the hard and uniform TiN coating, with a high work function, and the ability of the TiN coating to limit the undesirable effects of the underlying impurities and defects of the aluminum surface.

The surface finish of TiN-coated aluminum coupons, polished with 800 and 1200 grit silicon carbide paper was $\sim 7 \mathrm{~nm}$. This is smoother than electrode surfaces created using conventional polishing techniques at Jefferson Lab. The mechanical properties of the TiN electrode, hardness and modulus, were far superior to bare aluminum, and to stainless steel, niobium and titanium-alloy, based on literature. The hardness and modulus were measured as 18 and 270 GPa respectively. SEM microscopy scans of the residual impressions indicated no signs of delamination or peeling of the film from the substrate.

Aluminum electrodes, coated with TiN, could simplify the task of implementing photocathode cooling, which is required for future high current electron beam applications. For example, two designs for a future electron-ion collider $^{27,28}$ require electron guns that provide $\sim 100 \mathrm{~mA}$ average current to implement electron cooling of proton beams. The other accelerator applications, like the search for the proton electric dipole moment (pEDM), could benefit from TiN-coated aluminum electrodes. One experiment ${ }^{29,30}$ hopes to search for a proton EDM using spin-polarized and counterpropagating proton beams traveling inside an all-electric storage ring composed of hundreds of capacitor plates with a cumulative electrode path length of over $1 \mathrm{~km}$. Capacitor plates, made of aluminum and coated with TiN, could satisfy the electrostatic requirements of the storage ring, while costing considerably less than plates made of stainless steel. We speculate that the added cost of the TiN coating would be smaller 
than the cost savings associated with purchasing less expensive aluminum and significantly reduced labor charges for polishing. 


\section{Acknowledgments}

This material is based upon work supported by the U.S. Department of Energy, Office of Science, and Office of Nuclear Physics under contract DE-AC05-06OR23177. This work is authored by Jefferson Science Associates under U.S. DOE Contract No. DE-AC0584ER40150 and funded from the DOE Office of High Energy Physics and the Americas

Region ILC R\&D program. The U.S. Government retains a non-exclusive, paid-up, irrevocable, world-wide license to publish or reproduce this manuscript for U.S. Government purposes. 


\section{REFERENCES}

${ }^{1}$ R. V. Latham, High Voltage Vacuum Insulation, 2nd ed. (Academic Press, London, 1995), pp. 9, 38, 40.

${ }^{2}$ G. P. Beukema, Physica C 61, 259 (1972).

${ }^{3}$ D. W. Williams and W. T. Williams, J. Phys. D: Appl. Phys. 6, 734 (1973).

${ }^{4}$ E. Mahner, P. Kneisel, N. Pupeter, and G. Muller, Proc. of the 6th Workshop on Superconductivity, CEBAF, Newport News, Virginia, USA, October 4-8, 1993, p. 1085 .

${ }^{5}$ J. Francis, "SLC Procedural Note," FP-238-042-94 (1991) and modifications described in University of Illinois, Champaign, IL, USA (1991); B. Dunham , "Notes on Diamond Paste Polishing of Stainless Steel," Nuclear Physics Laboratory Tech Notes, 5/19/90 - revised 7/23/92, University of Washington, Seattle, WA, USA (1992).

${ }^{6}$ B. M. Dunham and A. C. Bartnik, et al., "Performance of the Cornell High-Brightness, High-Power Electron Injector” in: Proceedings of IPAC 2012, New Orleans, Louisiana, USA (May 2012).

${ }^{7}$ J. Maxson, I. Bazarov, B. Dunham, J. Dobbins, X. Liu, and K. Smolenski, Rev. Sci. Instrum. 85, 093306 (2014).

${ }^{8} \mathrm{M}$. BastaniNejad, Field emission studies toward improving the performance of DC high voltage photoelectron guns, Ph.D. Thesis, Old Dominion University, UMI publication number 3576662, USA (2013) p.49.

${ }^{9}$ M. BastaniNejad, A. A. Elmustafa, E. Forman, J. Clark, S. Covert, J. Hansknecht, C. Hernandez-Garcia, M. Poelker, L. Das, M. Kelley, P. Williams, "Evaluation of Electropolished Stainless Steel Electrodes for Use in DC High Voltage Photoelectron Guns," submitted for publication in JVST-A (in revision).

${ }^{10}$ M. BastaniNejad, M. A. Mohamed, A. A. Elmustafa, P. Adderley, J. Clark, S. Covert, J. Hansknecht, C. Hernandez-Garcia, M. Poelker, R. Mammei, and K. Surles-Law, Phys. Rev. ST Accel. Beams 15, 083502 (2012). 
${ }^{11}$ H. B. Michaelson, J. Appl. Phys. 48, 4729 (1977).

${ }^{12}$ H. Ahmadi and M. Nouri, J. Mater. Sci. 45, 3426 (2010).

${ }^{13}$ J. Westlinder, G. Sjöblom, and J. Olsson, Microelectron. Eng. 75 (4), 389 (2004).

${ }^{14}$ Engineering Properties of Steels, edited by P. D. Harvey (American Society for Metals, Metals Park, OH, 1982); D. Peckner and I. M. Bernstein, Handbook of Stainless Steels (McGraw-Hill Book Company, New York, NY, 1977); Metals Handbook, edited by H. E. Boyer and T. L. Gall (American Society for Metals, Materials Park, OH, 1985); Metals Handbook, ASM International Handbook Committee (ASM International, Materials Park, OH, 1990), 10 $0^{\text {th }}$ ed., vol. 1.

${ }^{15}$ Materials datasheet, Plansee USA LLC, 115 Constitution Boulevard Franklin, MA 02038, USA. http://www.plansee.com/en/Materials-13.htm.

${ }^{16}$ Materials Properties Handbook: Titanium Alloys, edited by R. Boyer, G. Welsch, and E. W. Collings (ASM International, Materials Park, OH, 1994); Metals Handbook, Properties and Selection: Nonferrous Alloys and Special-Purpose Materials (ASM International, 1990) $10^{\text {th }}$ ed., Vol. 2; Metals Handbook, Properties and Selection: Stainless Steels, Tool Materials and Special-Purpose Metals, ASM Handbook Committee (American Society for Metals, Materials Park, OH, 1980), $9^{\text {th }}$ ed., Vol. 3; J. M. Holt, H. Mindlin, and C. Y. Ho, Structural Alloys Handbook, edited by C. Y. Ho (CINDAS/Purdue University, West Lafayette, IN, 1996).

${ }^{17}$ Information provided by The Aluminum Association, Inc. from Aluminum Standards and Data 2000 and/or International Alloy Designations and Chemical Composition Limits for Wrought Aluminum and Wrought Aluminum Alloys (Revised 2001); Metals Handbook, Properties and Selection: Nonferrous Alloys and Special-Purpose Materials (ASM International, 1990) 10 ${ }^{\text {th }}$ ed., Vol. 2; Structural Alloys Handbook, edited by C. Y. Ho (CINDAS/Purdue University, West Lafayette, IN, 1996); Metals Handbook, edited by H. E. Boyer and T. L. Gall (American Society for Metals, Materials Park, OH, 1985).

${ }^{18}$ Nanoengineering of Structural, Functional and Smart Materials, edited by M. J. Schulz, A. D. Kelkar, and M. J. Sundaresan (CRC Press, Taylor \& Francis Group, 
Boca Raton, Florida, USA 2006), p. 535.

${ }^{19}$ Kawabata and T. Muto, Electrocomp. Sci. Tech. 8, 249 (1981).

${ }^{20}$ M. BastaniNejad, A. A. Elmustafa, E. Forman, J. Clark, S. Covert, J. Grames,J. Hansknecht, C. Hernandez-Garcia, M. Poelker, and R. Suleiman, Nucl. Instrum. Meth. A 762, 135 (2014).

${ }^{21}$ C. K. Sinclair, P. A. Adderley, B. M. Dunham, J. C. Hansknecht, P. Hartmann, M. Poelker, J. S. Price, P. M. Rutt, W. J. Schneider, and M. Steigerwald, Phys. Rev. ST Accel. Beams 10, 023501 (2007).

${ }^{22}$ Beam Alloy Technology LLC, 8270 Estates Pkwy, Plain City, OH 43064.

${ }^{23}$ K. Halbach, "A Program for Inversion of System Analysis and Its. Application to the Design of Magnets," Technical Report No. UCRL-17436 Lawrence Livermore National Laboratory, University of California , Livermore, CA, USA (1967).

${ }^{23}$ C. K. Sinclair, ICFA Newsletter 46, 97 (2008).

${ }^{24}$ W. C. Oliver and G. M. Pharr, J Mater Res. 19, 3 (2004).

${ }^{25}$ J. Hay, P. Agee, and E. Herbert, Exp. Tech. 34, 86 (2010).

${ }^{26}$ G. Hua and D. Li, Phys. Status Solidi B 249, 1517 (2012).

${ }^{27}$ E.C. Achenauer, M.D. Baker, A. Bazilevsky, et al., “eRHIC Design Study: An Electron-Ion Collider at BNL," arXiv:1409.1633 [physics.acc-ph] (2014).

${ }^{28}$ S. Abeyratne, A. Accardi, S. Ahmed, et al., "Science requirements and conceptual design for a polarized medium energy electron-ion collider at Jefferson lab," edited by Y. Zhang and J. Bisognano, arXiv:1209.0757 [physics.acc-ph] (2012).

${ }^{29}$ V. Anastassopoulos, S. Andrianov, R. Baartman, et al., "A Storage Ring Experiment to Detect a Proton Electric Dipole Moment,” arXiv:1502.04317 [physics.acc-ph].

${ }^{30}$ Y. K. Semertzidis, “A Storage Ring proton Electric Dipole Moment experiment: most sensitive experiment to CP-violation beyond the Standard Model," in Proceedings of the DPF-2011 Conference, Providence, RI, USA (August 8-13, 2011). 


\section{TABLES}

TABLE I.

\begin{tabular}{llllllc}
\hline \hline Material & $\begin{array}{l}\text { Work } \\
\text { function } \\
\phi(\mathrm{eV})\end{array}$ & $\begin{array}{l}\text { Thermal } \\
\text { conductivity } \\
(\mathrm{W} / \mathrm{m}-\mathrm{K})\end{array}$ & $\begin{array}{l}\text { Electrical } \\
\text { resistivity } \\
\mu \Omega-\mathrm{cm}\end{array}$ & $\begin{array}{l}\text { Hardness } \\
(\mathrm{GPa})\end{array}$ & $\begin{array}{l}\text { Elasticity } \\
\text { Modulus } \\
(\mathrm{GPa})\end{array}$ & $\begin{array}{l}\text { Density } \\
(\mathrm{g} / \mathrm{cc})\end{array}$ \\
\hline SS316L & $4.5^{10}$ & $16.3^{14}$ & $74^{3}$ & $1.5^{14}$ & $193^{14}$ & $8.0^{14}$ \\
Niobium & $4.3^{11}$ & $52^{15}$ & $14^{15}$ & $1.3^{15}$ & $104^{15}$ & $8.6^{15}$ \\
Molybdenum & $4.6^{11}$ & $142^{15}$ & $5.7^{15}$ & $2.2^{15}$ & $310^{15}$ & $10.9^{15}$ \\
Ti-6Al-4V & $4.5^{15}$ & $6.7^{16}$ & $178^{16}$ & $3.4^{16}$ & $114^{16}$ & $4.4^{16}$ \\
Al6061 & $3.5^{12}$ & $167^{17}$ & $4^{17}$ & $1.47^{*}$ & $70^{*}$ & $2.7^{17}$ \\
TiN coating & $5.0^{13}$ & $11-67^{18}$ & $14^{18}, 270^{19}$ & $18.2^{*}$ & $270^{*}$ & $3.3^{18}$ \\
\hline \hline
\end{tabular}

* Properties of IBED TiN coating on Al were measured in the present work

\section{TABLE II.}

\begin{tabular}{rrrrrrrr}
\hline \hline & A1 & A2 & TN1 & TN2 & TN3 & & \\
& $(\mathrm{He})$ & $(\mathrm{He})$ & $(\mathrm{He})$ & $(\mathrm{He})$ & $(\mathrm{He})$ & TA1 & TA2 \\
& $\begin{array}{r}1200 \\
\text { grit }\end{array}$ & $\begin{array}{r}1200 \\
\text { grit }\end{array}$ & 800 grit & grit & grit & DPP & DPP \\
& 9.0 & 9.1 & $>12.8$ & $>12.8$ & $>12.8$ & 10.9 & $>12.8$ \\
\hline $50 \mathrm{~mm}$ & 8.5 & 9.5 & $>13.7$ & $>13.7$ & $>13.7$ & 10.5 & $>13.7$ \\
$40 \mathrm{~mm}$ & 9.0 & 12.0 & $>15.4$ & $>15.1$ & 15.2 & 11.0 & $>15.4$ \\
$30 \mathrm{~mm}$ & 10.0 & 11.8 & 17.6 & 18.4 & 18.4 & 13.0 & $>18.7$ \\
\hline \hline $20 \mathrm{~mm}$ & & & & & & & \\
\hline \hline
\end{tabular}




\section{CAPTIONS}

TABLE I. Relevant characteristics of candidate electrode materials at room temperature.

TABLE II. The field strength required to produce $100 \mathrm{pA}$ of field emission, following helium gas conditioning, except titanium alloy sample TA2, which did not require it. For entries with (>) symbol, field emission current did not exceed $100 \mathrm{pA}$ at $-225 \mathrm{kV}$ bias voltage, the maximum voltage available.

FIG. 1 (Color online). (a) Schematic of the insulator, test electrode and anode used to collect the field emission. Photographs of the dc high voltage field emission test stands - (b) baked chamber TS1 and (c) unbaked chamber TS2 used to evaluate each cathode electrode.

FIG. 2 (Color online). Field emission current versus bias voltage and field strength for different cathode/anode gaps for (a) bare aluminum, (b) TiN-coated aluminum, and (c) titanium alloy electrodes. Labels with 'B' represent electrodes that are tested in the baked chamber TS1.

FIG. 3. SEM images of bare and TiN-coated aluminum electrode surface, (a) bare aluminum electrode surface showing a typical defect, (b) bare aluminum electrode surface showing a defect produced as a result of helium gas conditioning, (c) TiN-coated aluminum electrode surface showing a subsurface defect covered by TiN coating, and (d) TiN-coated electrode surface subjected to high field strength, and damaged postbreakdown.

FIG. 4 (Color online). Surface morphology of the TiN coated 1200 grit polished Al6061 coupon revealed by AFM with (a) 3D and (b) 2D displays. RMS roughness and average grain height were measured as 7 and $20 \mathrm{~nm}$ respectively for the scan area of $1 \mu \mathrm{m} \times 1 \mu \mathrm{m}$.

FIG. 5 (Color online). (a) Hardness and (b) Modulus as a function of contact depth of indentation for uncoated and TiN coated Al6061. The error bars represent 3 standard errors.

FIG. 6. SEM images of the residual impressions of (a) $500 \mathrm{~nm}$ and (b) $3 \mu \mathrm{m}$ Berkovich indents on the TiN coated 1200 grit polished Al6061 coupon. The occurrence of cracks for the larger indent was observed as pop-in events during indentation.

FIG. 7. Load versus displacement into surface for $2 \mu \mathrm{m}$ indents on an uncoated and TiN coated Al6061 coupons with 1200 grit surface finish. Pop-in events observed for indenter penetration depth of $\sim 500 \mathrm{~nm}$ and above which indicates fracture incidences. 

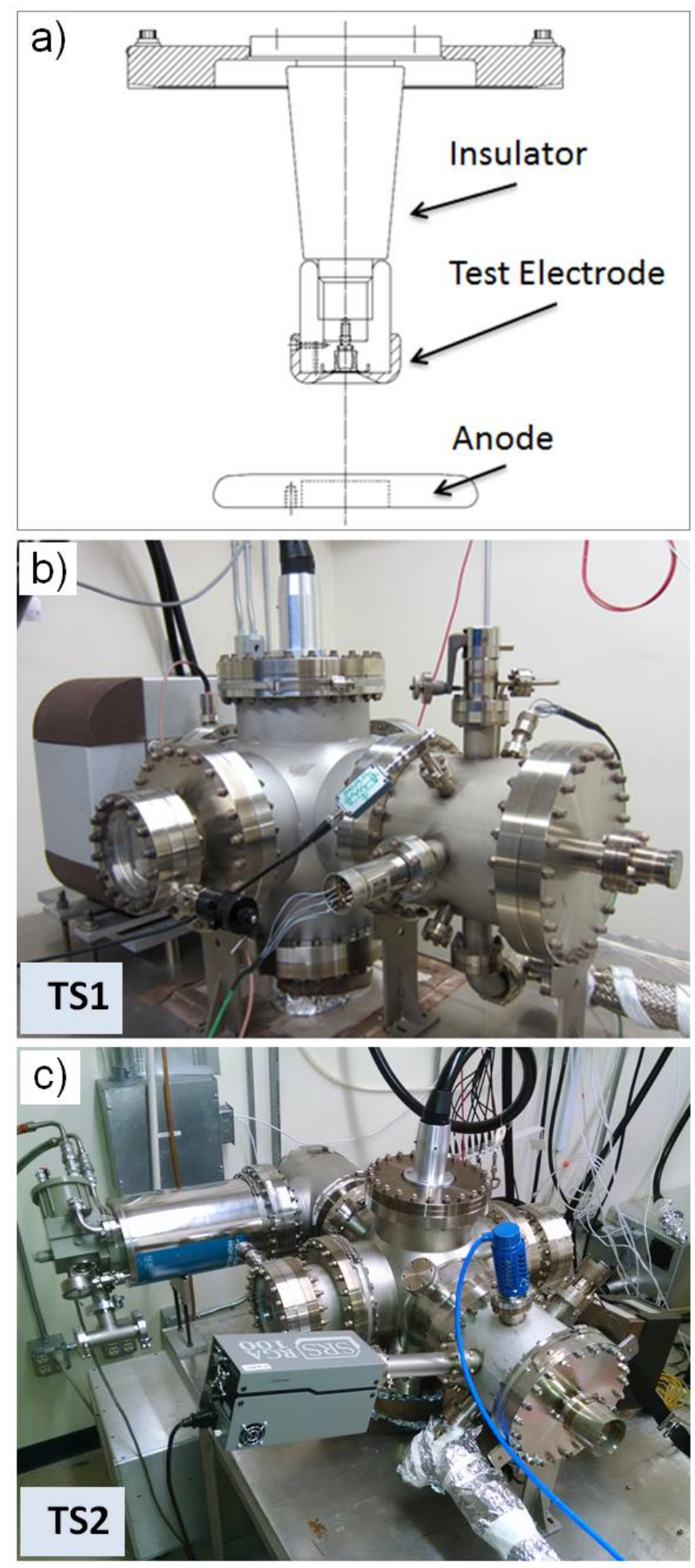

FIG. 1 (Color online). (a) Schematic of the insulator, test electrode and anode used to collect the field emission. Photographs of the dc high voltage field emission test stands - (b) baked chamber TS1 and (c) unbaked chamber TS2 used to evaluate each cathode electrode. 


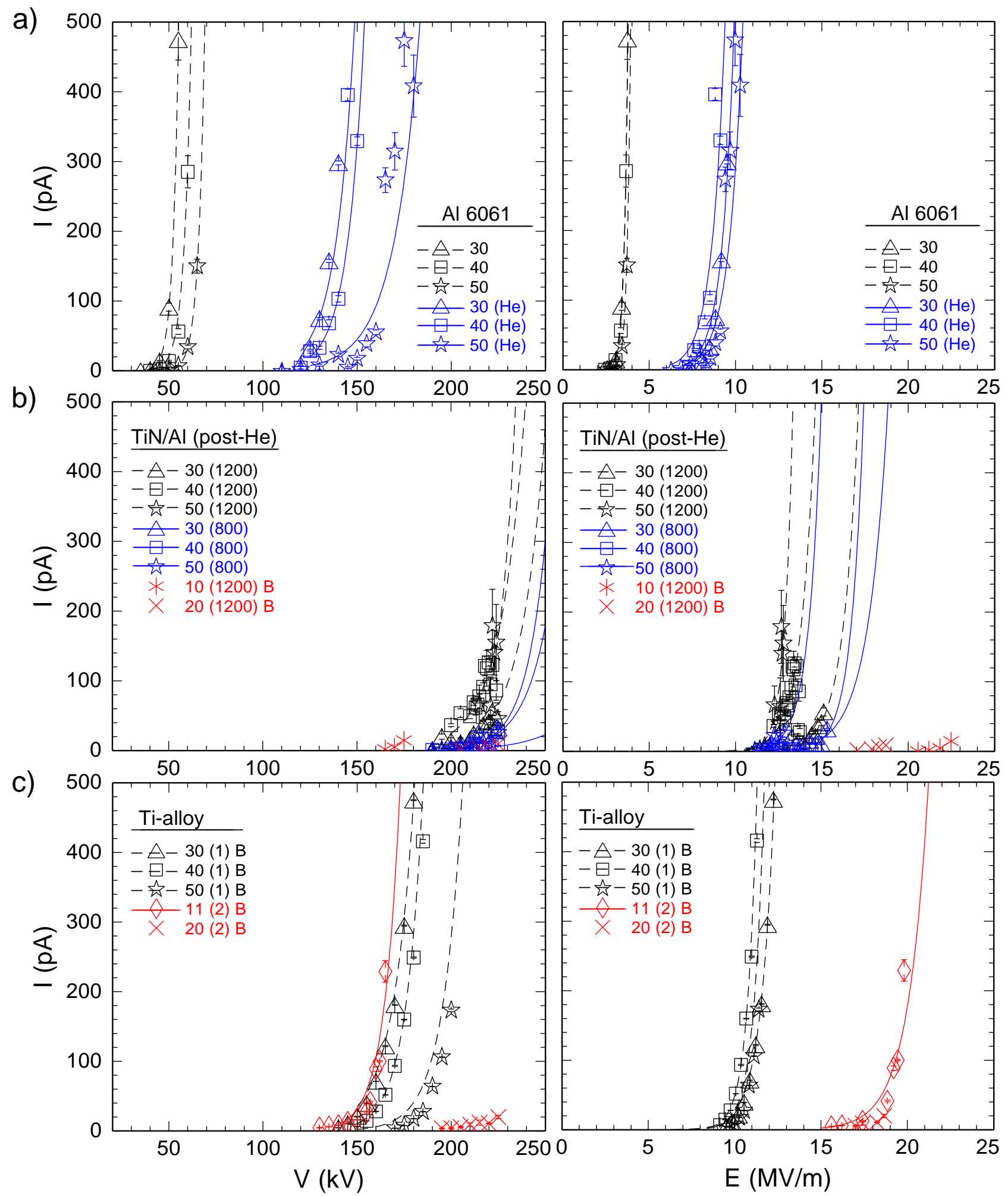

FIG. 2 (Color online). Field emission current versus bias voltage and field strength for different cathode/anode gaps for (a) bare aluminum, (b) TiN-coated aluminum, and (c) titanium alloy electrodes. Labels with 'B' represent electrodes that are tested in the baked chamber TS1. 

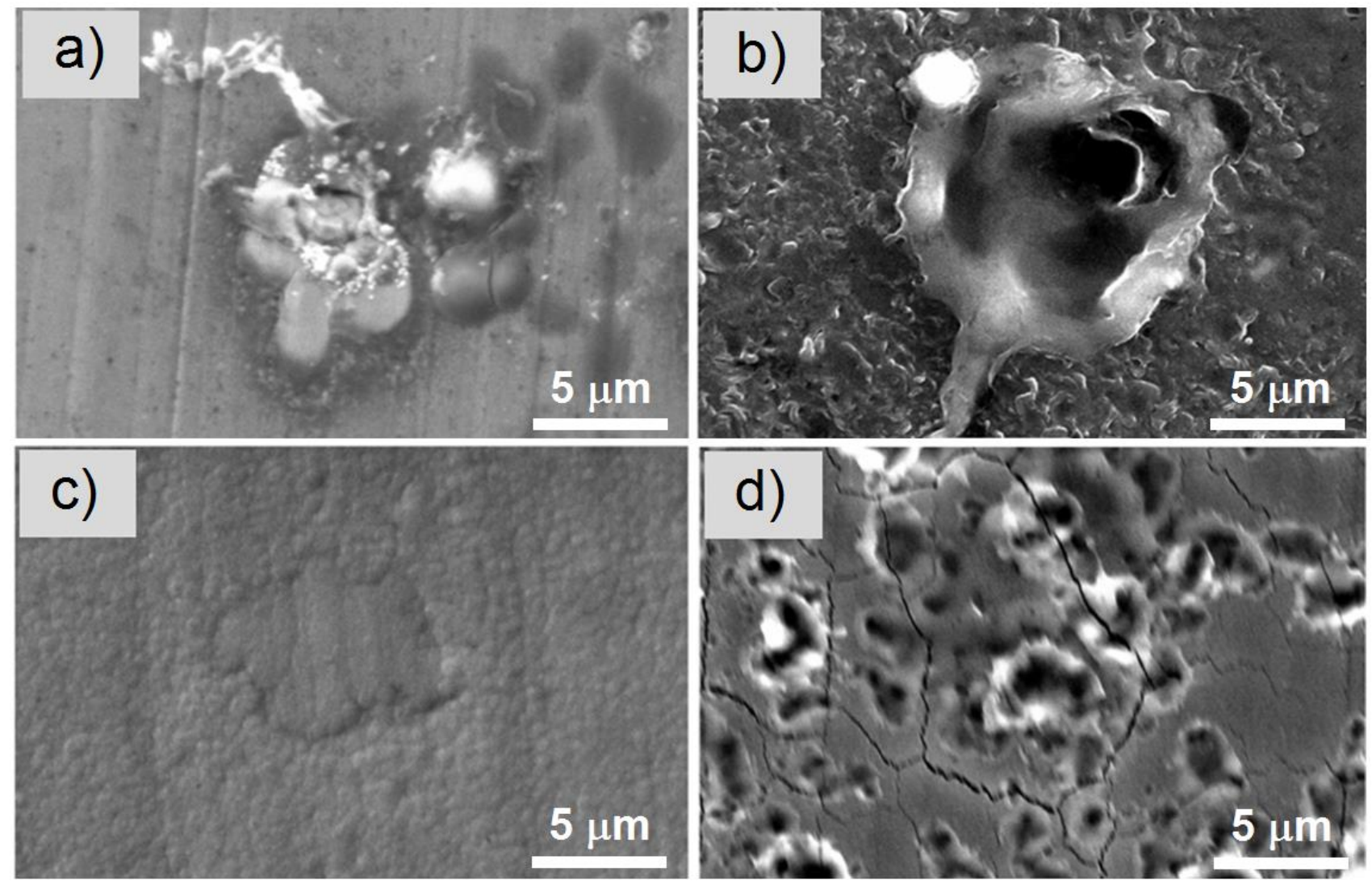

FIG. 3. SEM images of bare and TiN-coated aluminum electrode surface, (a) bare aluminum electrode surface showing a typical defect, (b) bare aluminum electrode surface showing a defect produced as a result of helium gas conditioning, (c) TiN-coated aluminum electrode surface showing a subsurface defect covered by TiN coating, and (d) TiN-coated electrode surface subjected to high field strength, and damaged post-breakdown. 


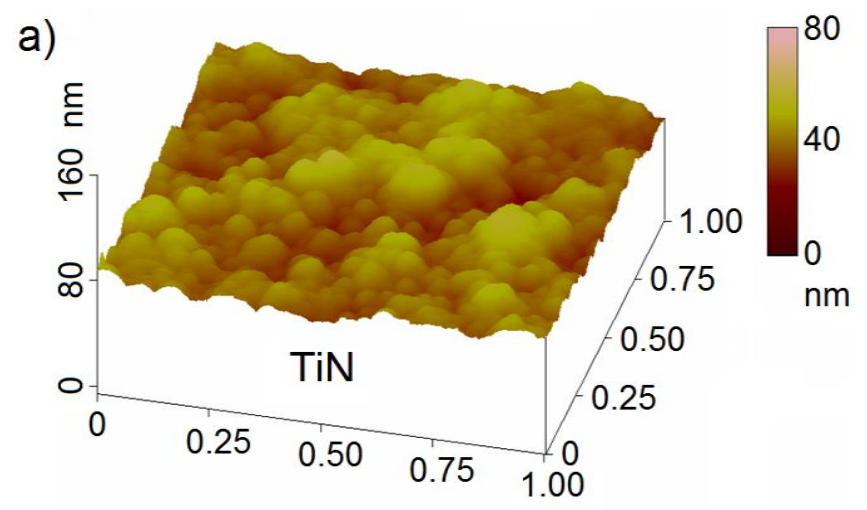

b)

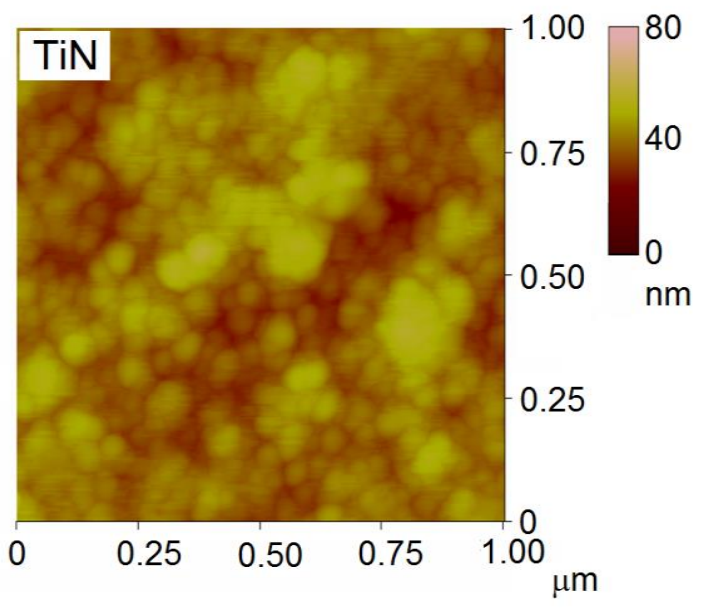

FIG. 4 (Color online). Surface morphology of the TiN coated 1200 grit polished Al6061 coupon revealed by AFM with (a) 3D and (b) 2D displays. RMS roughness and average grain height were measured as 7 and $20 \mathrm{~nm}$ respectively for the scan area of $1 \mu \mathrm{m} \times 1 \mu \mathrm{m}$. 
a)

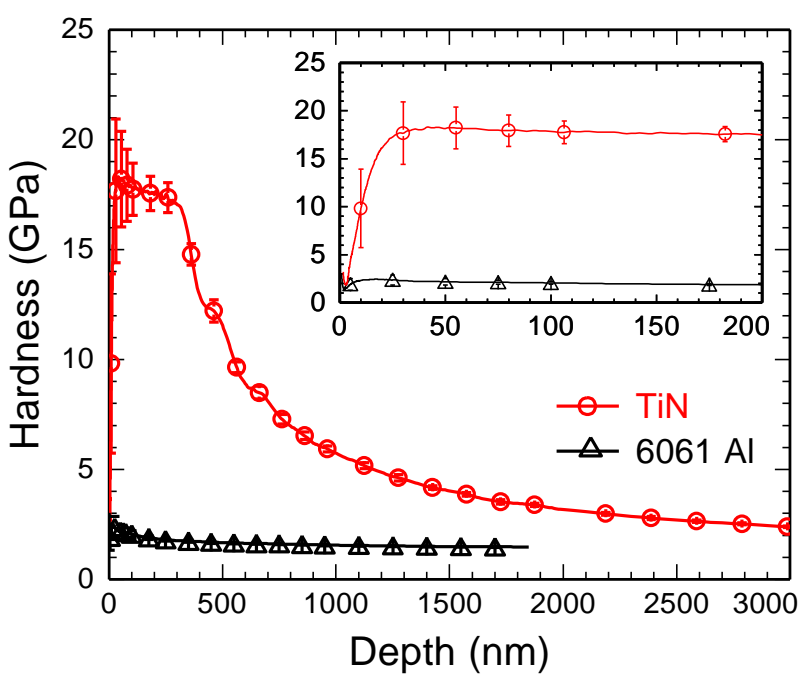

b)

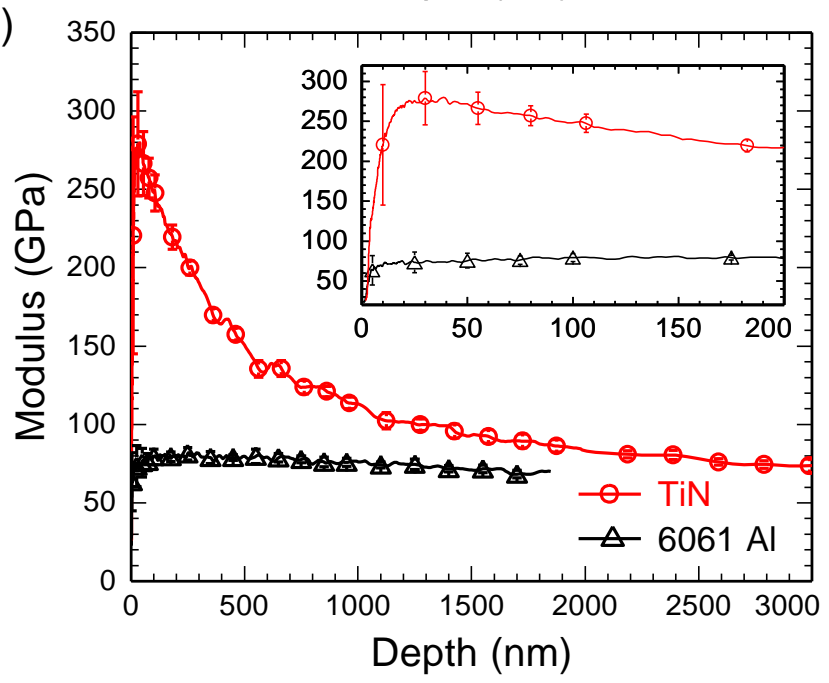

FIG. 5 (Color online). (a) Hardness and (b) Modulus as a function of contact depth of indentation for uncoated and TiN coated Al6061. The error bars represent 3 standard errors. 

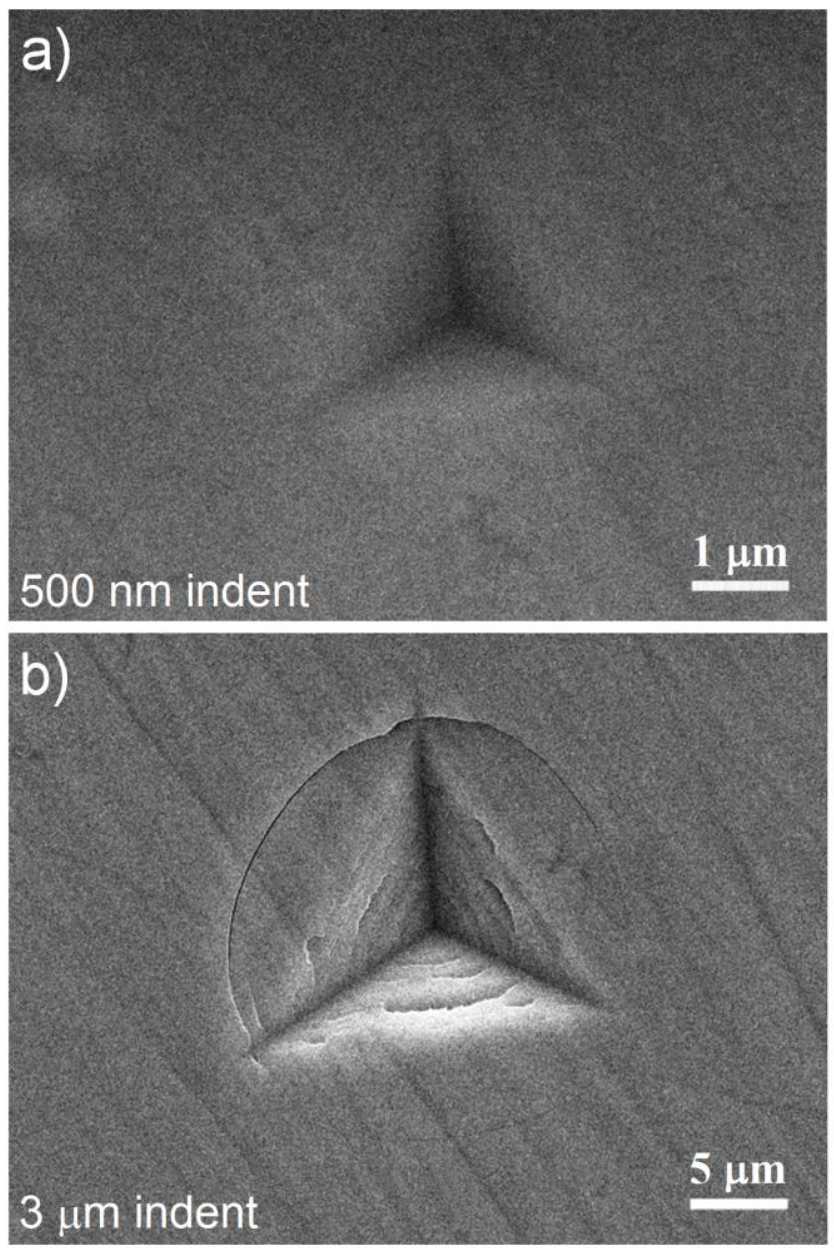

FIG. 6. SEM images of the residual impressions of (a) $500 \mathrm{~nm}$ and (b) $3 \mu \mathrm{m}$ Berkovich indents on the TiN coated 1200 grit polished Al6061 coupon. The occurrence of cracks for the larger indent was observed as pop-in events during indentation. 


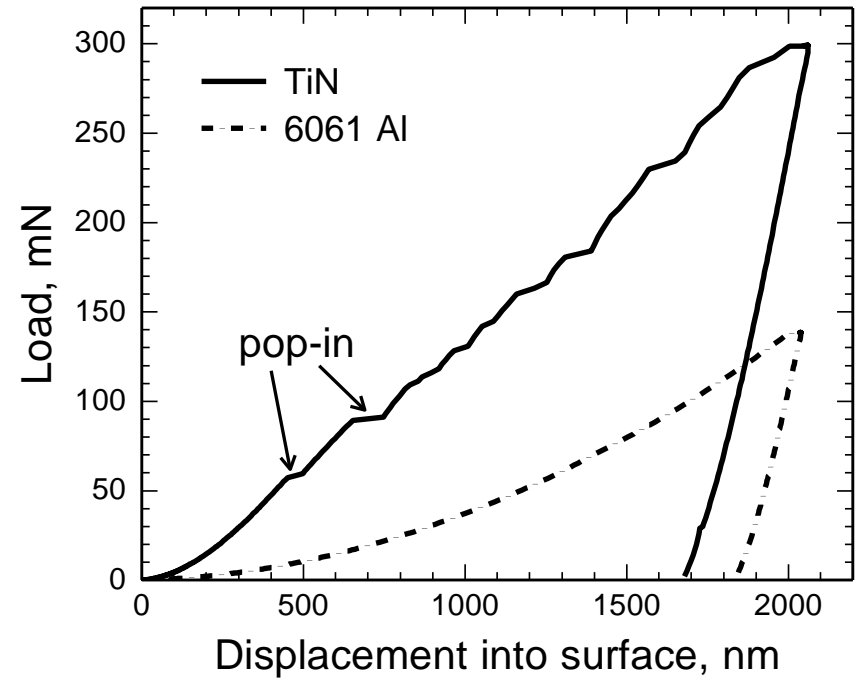

FIG. 7. Load versus displacement into surface for $2 \mu \mathrm{m}$ indents on an uncoated and TiN coated Al6061 coupons with 1200 grit surface finish. Pop-in events observed for indenter penetration depth of $\sim 500 \mathrm{~nm}$ and above which indicates fracture incidences. 